\title{
A!
}

This is an electronic reprint of the original article.

This reprint may differ from the original in pagination and typographic detail.

Välimäki, Salla; Beyeh, Ngong Kodiah; Linko, Veikko; Ras, Robin H.A.; Kostiainen, Mauri A.

\section{A supramolecular host-guest complex for heparin binding and sensing}

\section{Published in:}

Nanoscale

DOI:

$10.1039 / \mathrm{c} 8 \mathrm{nr} 03132 \mathrm{k}$

Published: 07/08/2018

Document Version

Peer reviewed version

Published under the following license:

Unspecified

Please cite the original version:

Välimäki, S., Beyeh, N. K., Linko, V., Ras, R. H. A., \& Kostiainen, M. A. (2018). A supramolecular host-guest complex for heparin binding and sensing. Nanoscale, 10(29), 14022-14030. https://doi.org/10.1039/c8nr03132k

This material is protected by copyright and other intellectual property rights, and duplication or sale of all or part of any of the repository collections is not permitted, except that material may be duplicated by you for your research use or educational purposes in electronic or print form. You must obtain permission for any other use. Electronic or print copies may not be offered, whether for sale or otherwise to anyone who is not an authorised user. 


\section{Supramolecular host-guest complex for heparin binding and sensing}

Salla Välimäki $i^{\mathrm{a}}$, Ngong Kodiah Beyeh ${ }^{\mathrm{b}, \mathrm{c}, \mathrm{d}}$, Veikko Linko ${ }^{\mathrm{a}}$, Robin H. A. Ras ${ }^{\mathrm{b}}$, Mauri A.

Kostiainen $^{\mathrm{a}, \mathrm{e}^{*}}$

${ }^{a}$ Biohybrid Materials, Department of Bioproducts and Biosystems, Aalto University, P.O. Box 16100, FI-00076 Aalto, Espoo, Finland

${ }^{\mathrm{b}}$ Soft Matter and Wetting, Department of Applied Physics, Aalto University, P.O. Box 15100, FI-00076 Aalto, Espoo, Finland

${ }^{\mathrm{c}}$ University of Windsor, Department of Chemistry and Biochemistry, Windsor, ON N9B 3P4, Canada

${ }^{\mathrm{d}}$ Department of Chemistry, Oakland University, 146 Library Drive, Rochester, Michigan 483094479, USA

${ }^{\mathrm{e}}$ HYBER Centre of Excellence, Department of Applied Physics, Aalto University, FI-00076 Aalto, Finland 


\begin{abstract}
Heparin is an anionic polysaccharide widely used in clinics as an anticoagulant. However, heparin usage requires an antidote and sensors for safe operation during and after surgeries. In this study, a host-guest complex capable in selective heparin binding and sensing is presented. Heparin binding affinity was studied in solution with a variety of polycationic macrocyclic hosts, a pillar[5]arene and multiple resorcin[4]arenes, by dynamic light scattering, dye displacement assay, isothermal titration calorimetry, and anti-Xa assay. The measurements reveal the significant importance of multivalency in the electrostatic host-heparin binding in competitive, application-relevant media. Additionally, to monitor heparin concentration, a host-guest indicator displacement assay was performed by following the free and bound state of methyl orange dye in $\mathrm{UV}-\mathrm{V}$ is spectroscopic experiments. Furthermore, this colorimetric sensing based on the tertiary host-guest-heparin supramolecular assembly was utilized in the construction of a calibration curve in a range of blood plasma concentrations.
\end{abstract}

\title{
Introduction
}

Heparin is a naturally occurring highly charged polyanion and a well-known anticoagulant widely used in surgical practice. ${ }^{1}$ More specifically, it is a negatively charged sulfated polysaccharide belonging to the group of glycosaminoglycans (Scheme 1). The anticoagulant effect of heparin is based on its ability to activate antithrombin-III, which, subsequently, inactivates vital coagulation cascade substance such as thrombin and factor Xa consequently preventing fibrin and clot formation. ${ }^{2}$ To balance and neutralize heparin dosing, a heparin 
inhibitor is needed, and commonly, arginine-rich protein protamine sulfate (PS) is used for this purpose ${ }^{3,4}$ However, in recent years there has been an interest to investigate alternatives for protamine due to its adverse effects like hypotension and serious allergic reactions. ${ }^{5-7}$ In the neutralization process, electrostatic interactions play a key role as the heparin-protamine complex is formed due to the attractive forces rising from heparin's negative and protamine's positive charge. In addition to neutralization methods, also sensing applications during and after surgery are required for heparin concentration determination. ${ }^{3}$ Commonly, activated partial thromboplastin time (aPTT) technique and anti-Xa assay are used. ${ }^{8}$ However, these currently available analysis methods have certain limitations including preanalytical and postanalytical variabilities $^{8,9}$ leading to the need of improved heparin sensing systems.

Many colorimetric ${ }^{10-14}$ and fluorescent ${ }^{15-30}$ heparin sensors with different functionalities and varying structures from small molecules ${ }^{11,14,18-22}$ and host-guest complexes ${ }^{16,24}$ to synthetic polymers $^{12,27-29}$ and peptides ${ }^{31,32}$ have been developed. For instance, a boronic acid based receptor utilizing preorganization of the structure provided a colorimetric heparin indication system capable in selective heparin binding. ${ }^{11}$ Amine and guanidine functional groups, on the other hand, were exploited in the development of self-assembling fluorescent receptors designed for electrostatic heparin binding. ${ }^{24}$ In this system, a variety of cyclodextrin hosts and fluorescent guests were used to detect the purity of heparin. Additionally, induced fit of cationic calix[8]arenes has been used for heparin binding with improved complexation when compared to protamine. ${ }^{16}$ This system also provides signaling that is based on indicator displacement monitored with fluorescence spectroscopy. 
Inspired by these macrocyclic heparin sensors, we have designed a series of cationic macrocycles capable of heparin binding in addition to host-guest binding. In more detail, we have studied resorcin[4]arenes and pillar[5]arene with varying number of positive charges and different cavity sizes (Scheme 1). Resorcinarenes, obtained from an acid catalyzed condensation between resorcinol and aldehydes, are well-known macrocyclic host systems for a wide variety of guests. ${ }^{33}$ Pillararenes, on the other hand, are synthetized by linking hydroquinone ethers with methylene bridges to form a rigid rings. ${ }^{34}$ Both of these macrocyclic receptors can be modified by attaching different functional groups at the lower and upper rims, and therefore these receptors have been developed for many applications, for example, in the areas of biomedicine $\mathrm{e}^{35,36}$ and material science ${ }^{34,37}$. The resorcin[4]arenes used here exists in their $\mathrm{C}_{4 v} \mathrm{v}$ crown conformation which is locked by intramolecular hydrogen bonds between adjacent hydroxyl groups at the upper rim. Further functionalization of the upper rim with amine containing moieties in a Mannich ${ }^{38,39}$ condensation reaction with excess formaldehyde and subsequent ring opening with mineral acids ${ }^{40}$ give the resorcin[4] arenes a cationic character that enables binding with anionic heparin. The pillar[5]arene used in this study has a characteristic pillar-like structure and cationic moieties on both upper and lower rim in addition to an electronrich internal cavity accessible from both sides. ${ }^{41}$ By increasing the number of charges in the host structures from +4 to +16 , enhanced heparin binding is expected due to multivalency. An anionic resorcin[4]arene not expected to interact with heparin was included in the research as a control compound. ${ }^{42}$

Binding affinity between heparin and the resorcin[4]arenes (R4-, R4+, R8+ and $\mathbf{R} 16+)$ as well as the pillar[5]arene $(\mathbf{P 1 0}+)$ was studied in solution with dynamic light scattering (DLS), 
methylene blue (MB) displacement assay, isothermal titration calorimetry (ITC), and anti-Xaassay. With these methods, we verified that binding affinity towards heparin indeed increases with increasing number of charges on the macrocycles. However, the resorcin[4]arene with four protonated amines did not show any complexation with heparin in the physiologically relevant conditions and concentration ranges used in this study. As assumed, the anionic resorcinarene did not have any affinity towards heparin.

In addition to the heparin binding, the host-guest system was able to detect heparin levels in application-relevant media. For this purpose, the most powerful host-guest combination is methyl orange (MO) as the guest, and P10+ as the host in 1:10 MO-P10+ molar ratio. The detection is based on ratiometric sensing by monitoring absorption maxima of free and bound MO. Moreover, a calibration curve for quantitative heparin dose detection in varying blood plasma contents was constructed. 


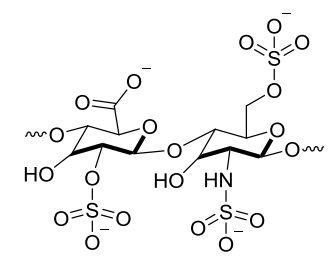

heparin

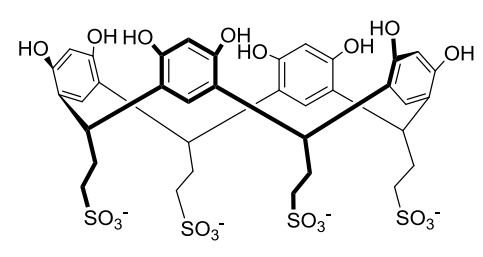

R4-

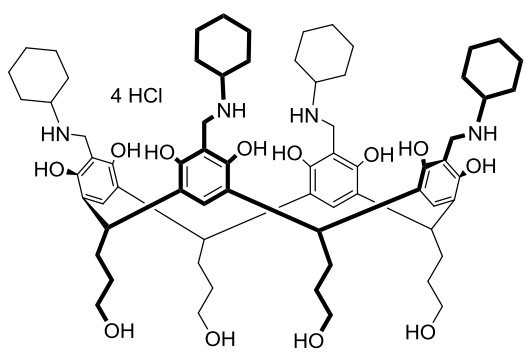

R4+

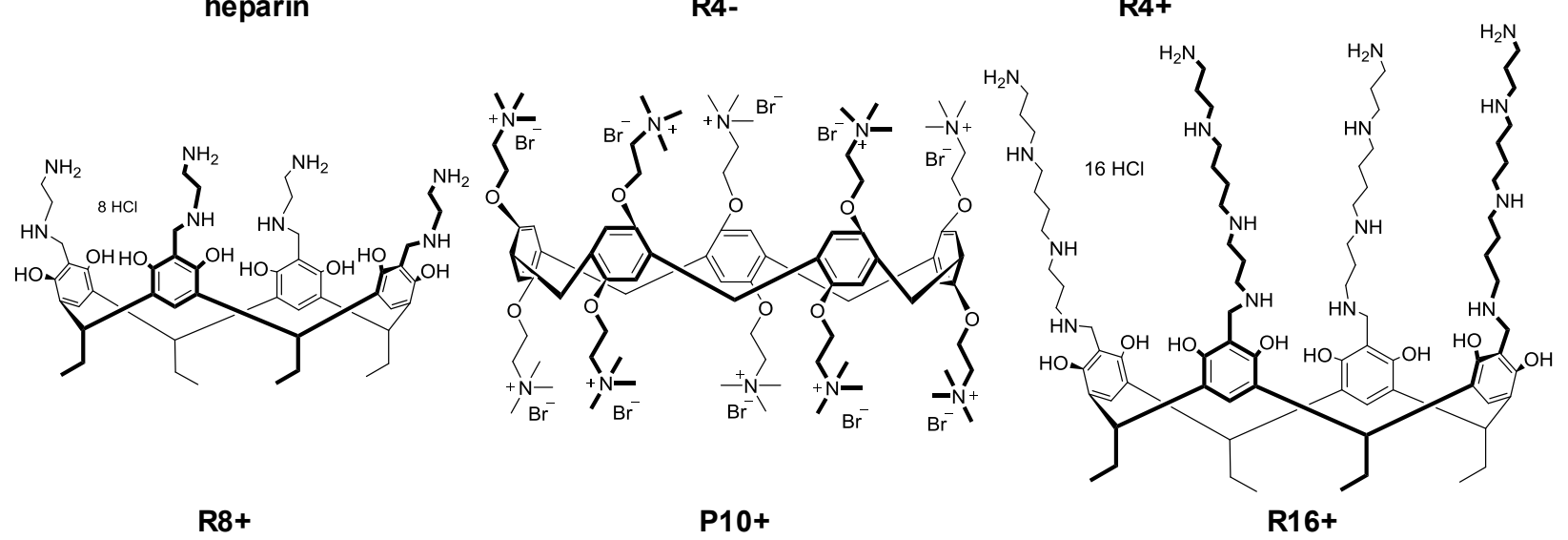

Scheme 1. Structure of major repeating unit of heparin and macrocyclic hosts used in the study.

Hosts are named based on their structure ( $P=$ pillar[5]arene, $R=$ resorcin[4]arene) and nominal number of charges.

\section{Results and Discussion}

Binding between the hosts and heparin was investigated through a series of DLS experiments. In the measurements, heparin solution was titrated with host solution and hydrodynamic diameter and count rate were monitored. As shown in Figure 1a, P10+ and protamine clearly display increasing count rate up to host-to-heparin mass ratio of 1.5 indicating heparin-host complex formation. Similar behavior was observed also for $\mathbf{R 1 6 +}$ and $\mathbf{R 8}+$ only with lower count rates. Therefore, based on the count rate data, optimal mass ratio for complex formation ranges between 1.25 and 1.5. In binding between $\mathbf{P 1 0}+$ and heparin, these values correspond in to 
neutral charge balance, which is a well-known phenomenon in complex formation between oppositely charged polyelectrolytes. ${ }^{43,44}$ On the contrary, hosts $\mathbf{R} 16+$ and $\mathbf{R 8}+$ need a charge excess for full complexation of heparin. This difference might be due to better availability of binding sites in the bimodal structure of $\mathbf{P 1 0}+$ when compared to unimodal nature of the cationic groups in $\mathbf{R 1 6 +}$ and $\mathbf{R 8 +}$ (Scheme 1).

When the hydrodynamic diameter data is examined in detail, it is obvious that R16+ and R8+ form the largest complexes with heparin whereas P10+ and protamine yield smaller host-heparin complexes (Figure 1b). In previous studies, a structure- and concentration-dependent behavior of macrocycle-polyanion complexation has been observed. Therein, it was concluded that the long alkyl chains on the lower rim of the amphiphilic receptors induce formation of smaller complexes of 50-60 nm. ${ }^{45,46}$ However, our resorcin[4]arenes lack these long alkyl chains leading to the increase in complex sizes. Protamine and $\mathbf{P 1 0 +}$, on the contrary, have no hydrophobic tails, meaning that only electrostatic interactions are affecting the binding process. For further confirmation of the complexation, all of these compounds (protamine, $\mathbf{R 1 6}+, \mathbf{P 1 0}+$ and $\mathbf{R 8}+$ ) presented visually observable phase separation upon titration.

In both count rate and hydrodynamic diameter data, standard deviation values indicate that complex size and complex formation suffer from fluctuation. However, in signaling systems, large complex size and variation of the size are not destructive as long as they do not affect signaling accuracy. 
Based on the DLS data of R4+ and R4-, no complexation occurs between heparin and these resorcinarenes. For $\mathbf{R 4 +}+$, insignificant count rates and hydrodynamic diameters were measured but no confirmation of complex formation was detected even at high mass and charge ratios. This is most likely due to the low number of amine groups and steric hindrance caused by the cyclohexane groups in the upper rim functionality. As expected, no presentable data for R4- was obtained as the measurements had too low count rates to produce reliable size data, making this receptor a good control compound. Additionally, neither of the titrations between heparin and hosts (R4+ and $\mathbf{R 4 - )}$ resulted to any increase in turbidity of the sample.

DLS studies of host molecule P10+ were carried out also by including the guest molecule, methyl orange, into the system. In these measurements, no significant difference was observed when compared to the results without MO (Figure S1) indicating that host-guest binding does not interfere with the heparin-host complex formation.

From these measurements, it can be concluded that heparin binding is evident for hosts $\mathbf{R} 16+$, $\mathbf{P 1 0 +}$ and $\mathbf{R 8 +}$, and it is as effective as for protamine. Additionally, binding is concentration-and structure-dependent, and is not affected by the host-guest complex formation.

Table 1. Heparin and host binding parameters at point of optimal binding based on DLS count rate data. Mass, molar and charge ratios (host:heparin) are presented. Charge ratios are calculated assuming that all primary, secondary and tertiary amines carry one positive charge and heparin with molecular weight of $18 \mathrm{kDa}$ has 90 charges $^{47}$. 


\begin{tabular}{cccc}
\hline host & mass ratio & molar ratio & charge ratio \\
\hline R16+ & 1.25 & 11.07 & 1.97 \\
P10+ & 1.50 & 11.89 & 1.32 \\
R8+ & 1.50 & 22.87 & 2.03
\end{tabular}

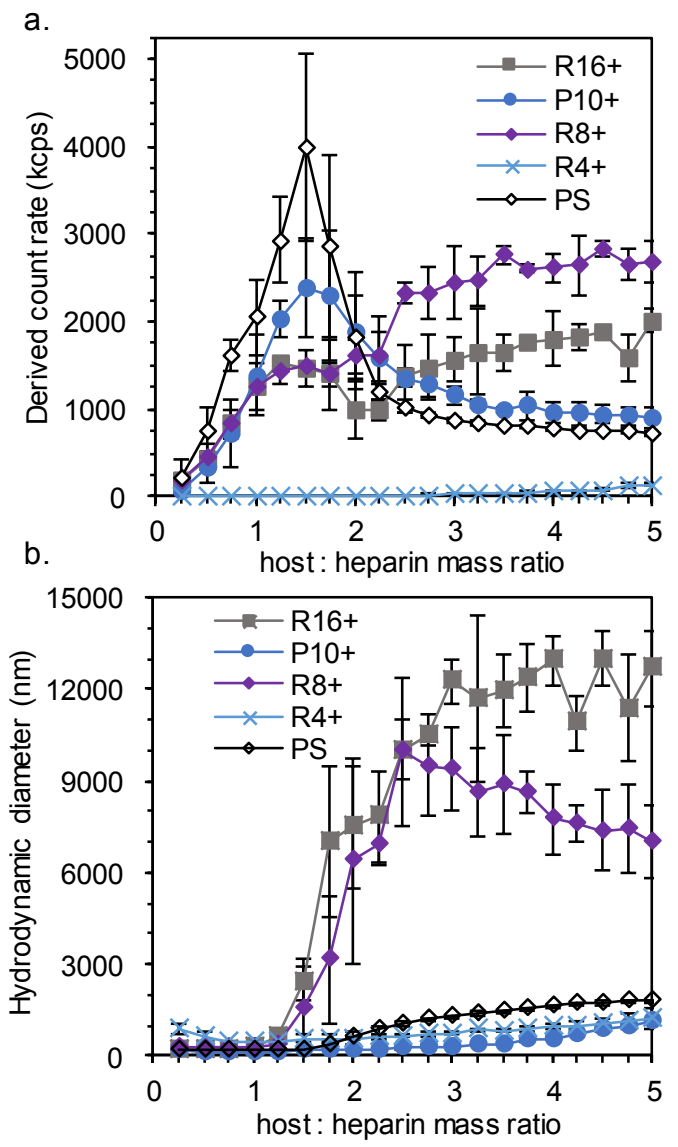

Figure 1. DLS data confirms complex formation between heparin and all the host molecules, except $\boldsymbol{R} 4+$ and $\boldsymbol{R} 4-$, in physiologically relevant buffer (10 mM Tris- $\mathrm{HCl}, 150 \mathrm{mM} \mathrm{NaCl}$, pH 7.4). a) Count rate is highest at host:heparin mass ratio $1.25-1.5$ for $\boldsymbol{R} \mathbf{1 6}+, \boldsymbol{P 1 0}+, \boldsymbol{R} \boldsymbol{8}+$ and protamine sulfate (PS). b) Largest complexes are observed for $\mathbf{R} \mathbf{1 6}+$ and $\mathbf{R} \mathbf{8}+$ and smaller ones for P10+ and protamine. Titrations were carried out in triplicates and averages with standard deviation shown. 
Heparin neutralization efficiency of the host molecules was also studied with methylene blue displacement assay in which heparin-MB mixture was titrated with the host solution. Upon increasing host concentration, absorbance maximum shifts from $568 \mathrm{~nm}$ to $664 \mathrm{~nm}$ if methylene blue in the heparin-MB complex is released and replaced with the competing host. This kind of behavior was observed for host molecules R16+, P10+ and R8+ (Figure 2), which is in good agreement with the DLS data. Especially $\mathbf{R} 16+$ and $\mathbf{P 1 0}+$ have promising complexation profiles when compared to protamine, as they are almost equally efficient in heparin neutralization. Furthermore, based on the Table 1, it seems that P10+ is even more efficient than R16+ as lower charge excess is needed with P10+. As was concluded also from the DLS data, this might be due to the better accessibility of the P10+ charges as they are located on both sides of the molecules versus unimodal localization of the cationic groups on the resorcinarenes. Moreover, R8+ shows complexation with heparin even though it is not as efficient as with $\mathbf{R 1 6 +}, \mathbf{P 1 0}+$ and protamine. As observed also in the DLS measurements, R4+ shows no complexation with heparin in the concentration ranges studied here. This can be attributed again to lower number of charges when compared to the other hosts, steric hindrance caused by the cyclohexyl group in the R4+ structure or due to lower binding affinity when compared to MB. Additionally, as expected, negatively charged R4- does not show any complexation with the anionic heparin. Moreover, measurements with host P10+ were carried out also together with the guest molecule, methyl orange. As in the DLS measurements, MO did not interfere with the host-heparin binding (Figure S2).

From these results, it can be concluded that $\mathbf{R 1 6}+$ and $\mathbf{P 1 0}+$ are the most promising alternatives for heparin binding and sensing. Overall, based on the differences observed here and in the 
previous studies on the electrostatic binding of heparin ${ }^{16,18}$, multivalency has a high importance in the competitive heparin binding.

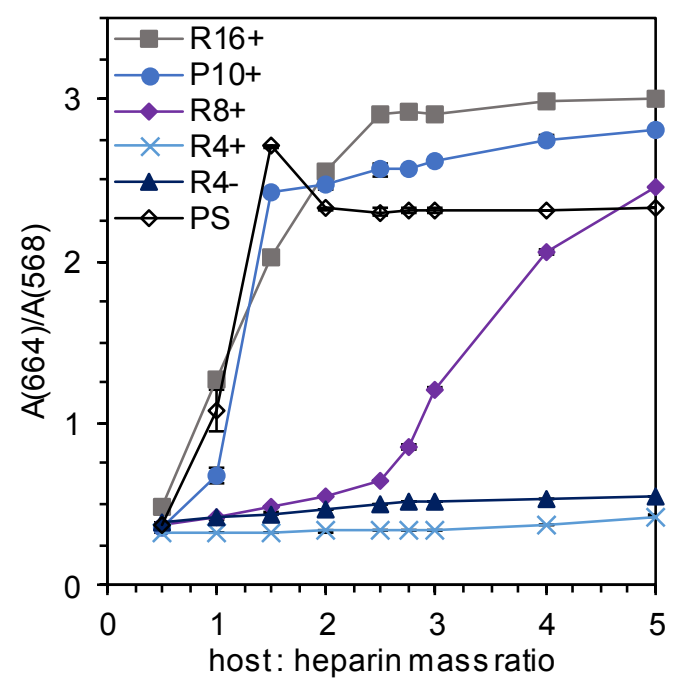

Figure 2. Methylene blue displacement assay confirms concentration-dependent complex formation between heparin and hosts $\mathbf{R 1 6 + , ~ P 1 0 + , ~} \mathbf{R} 8+$ in $10 \mathrm{mM}$ Tris-HCl, $\mathrm{pH}$ 7.4. Protamine (PS) is shown as a reference. Hosts $\boldsymbol{R} 4+$ and $\boldsymbol{R} 4-$ are not able to displace MB. Assay was performed three times and averages with standard deviation shown.

ITC measurements were conducted to further understand the thermodynamics of the host-heparin binding. The calorimetric titration plot obtained for P10+ with heparin (Figure 3, Table S1) indicates efficient binding between P10+ and heparin and after background reduction results can be fitted with a sequential binding mode. The system corresponds roughly to binding saturation at molar ratio of seven, which is in relatively good agreement with the 1:1 charge ratio observed also in the DLS data. The binding is enthalpy and entropy driven and spontaneous at $298 \mathrm{~K}$. The first six binding interactions are very strong where the highest binding constant $(\mathrm{K})$ is $2.75 \times 10^{5}$ $\mathrm{M}^{-1}$ and the lowest $6.39 \times 10^{4} \mathrm{M}^{-1}$. The last binding before saturation is relatively weak with a 
binding constant of $1.29 \times 10^{2} \mathrm{M}^{-1}$. Additionally, binding between R16+ and heparin follows the same trend but with less R16+ molecules per heparin molecule as the stoichiometry is approximately six (Figure S3a and Table S2). All the binding sites indicate spontaneous binding and overall the binding is both entropy and enthalpy driven. The binding constants fluctuate but stay relatively strong between $1.11 \times 10^{3}$ and $6.31 \times 10^{5} \mathrm{M}^{-1}$. Further, for $\mathbf{R 8}+$, the stoichiometry dropped to four molecules per one heparin molecule and the spontaneous binding showed lower binding constants $\left(1.25 \times 10^{3}-1.95 \times 10^{4} \mathrm{M}^{-1}\right)$ compared to the other hosts (Figure S3b and Table S3). As with the other host molecules, the also R8+ showed both entropy and enthalpy driven interactions with heparin. Based on the results of the ITC measurements, we can further confirm the electrostatic binding between heparin and the host molecules observed also in the DLS and MB displacement assay experiments. 


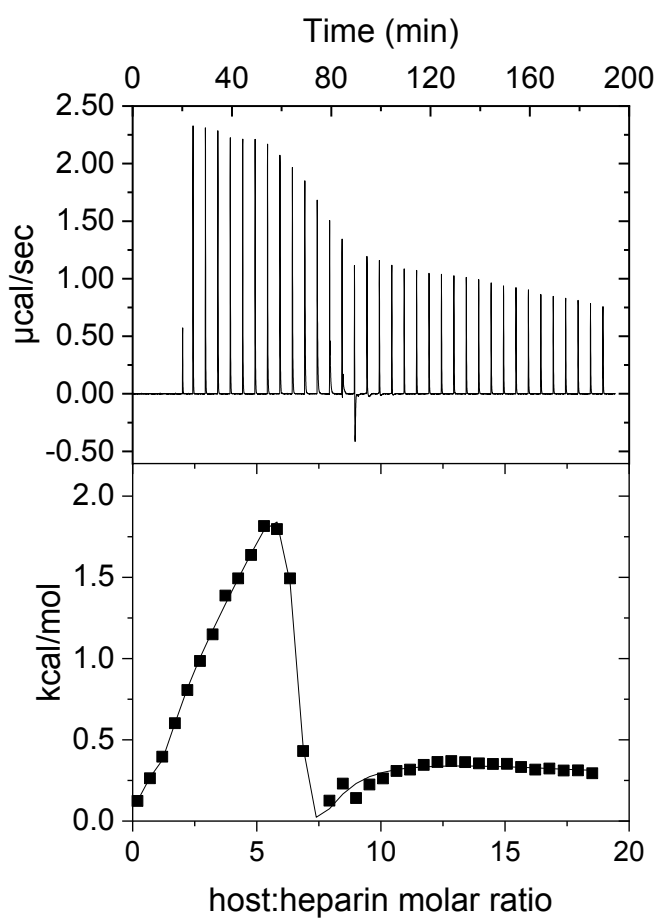

Figure 3. ITC data of the titration of host P10+ $(5 \mathrm{mM})$ into heparin $(0.05 \mathrm{mM})$ in $10 \mathrm{mM}$ TrisHCl buffer pH 7.4 at $298 \mathrm{~K}$. Upper graph showing the raw data and the lower graph presenting the integrated data fitted into a sequential binding model after background reduction.

In vitro heparin neutralization efficiency of the hosts was studied and compared to protamine by using a chromogenic 2-stage anti-Xa assay. In Figure 4, it can be seen that neutralization effectiveness is dependent on the multivalency and concentration of the hosts. For instance, hosts $\mathbf{P 1 0}+$ and $\mathbf{R 1 6}+$ are relatively efficient in heparin neutralization, and increasing the host concentration enhances the neutralization up to $98 \%$ for $\mathbf{P 1 0}+$. On the contrary, $\mathbf{R 8}+$ does not show any efficiency in these concentrations, which is in line with the results from the MB displacement assay measurements. This lower binding efficiency even at high $\mathbf{R 8 +}$ concentrations is due to the lower number of charges that plays a key role especially in 
competitive media such as in blood plasma. Hosts R4+ and R4- do not show any neutralization effect (Figure S5) as was concluded also from the DLS measurements and MB displacement assay experiments. Similar effect of concentration and number of charges on neutralization efficiency has been detected also previously with polymeric heparin binders. ${ }^{48-50}$ In short, the anti-Xa assay confirms that heparin neutralization is efficient and linearly dose-dependent with hosts P10+ and R16+. Moreover, at higher host concentrations, full neutralization of heparin is essentially achieved with P10+.

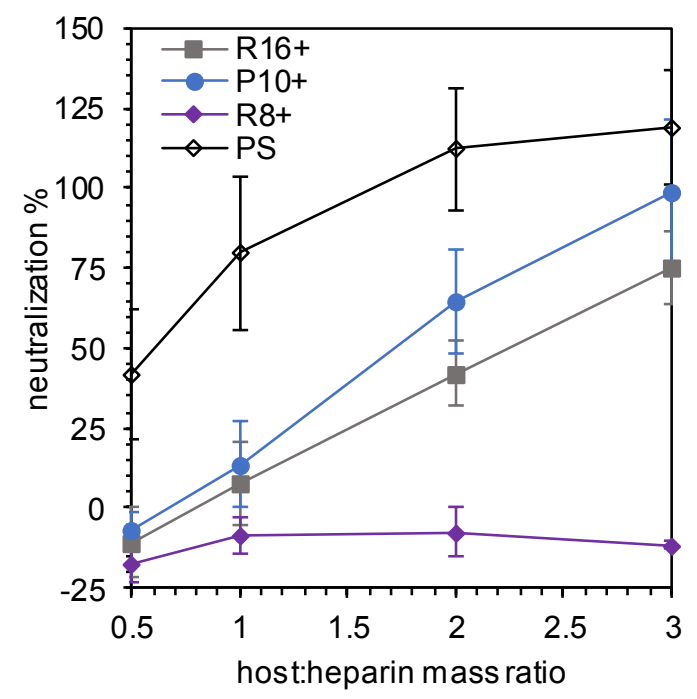

Figure 4. Anti-Xa assay performed in plasma proves that hosts $\mathbf{R} \mathbf{1 6}+$ and $\mathbf{P 1 0}+$ are effective heparin neutralizers in application-relevant media, and at high concentrations they show activity comparable to the currently used heparin antidote, protamine sulfate (PS). On the contrary, $\mathbf{R} 8+$ is not effective in the concentration ranges used here. Assay was performed in triplicates and averages with standard deviation shown. 
Host-guest binding between the cationic hosts and methyl orange was studied with UV-Vis spectroscopy by comparing absorption maxima corresponding to free $(470 \mathrm{~nm})$ and bound (405 $\mathrm{nm}$ ) state of $\mathrm{MO}$ (Scheme 2). In figure 5a, a clear decrease in $\mathrm{A}(470) / \mathrm{A}(405)$ ratio can be detected for host P10+ indicating that it is the most effective receptor for MO. This data is derived from the original absorption spectra presented in Figure $5 \mathrm{~b}$ where a clear blue shift in the spectra is observed. Also hosts $\mathbf{R 1 6 +} \mathbf{R 8}^{+}$and $\mathbf{R 4 +}$ are able to bind the guest to some extent as can be concluded from the slight decrease of $\mathrm{A}(470) / \mathrm{A}(405)$ ratio in Figure 5a (original absorption spectra for all resorcin[4]arenes is presented in supporting information Figure S6). However, the spectral changes are insignificant when compared to P10+. The decreased guest binding affinity of the resorcin[4]arene hosts is most probably due to their smaller cavity size and less complementary structure. Additionally, resorcinarenes used in this study exist in crown conformation due to the intramolecular hydrogen bonds of the upper rim hydroxyl groups thus leading to shallow cavities. The tube-like cavity of the bimodal pillar[5]arene is larger and more complementary to the rod-like MO. ${ }^{51-54}$ Protamine was used as a control and no changes in the MO spectra were observed indicating that protamine does not bind MO (data not shown).

Host-guest binding between $\mathrm{MO}$ and hosts $\mathbf{R 8}+, \mathbf{P 1 0}+$ and $\mathbf{R 1 6}+$ were additionally investigated with ITC (Figure S4) at $298 \mathrm{~K}$. The binding between host P10+ and MO was further confirmed from these ITC analysis, which reveals the complexation to be exothermic, enthalpy driven and spontaneous $(\Delta \mathrm{H}<0, \mathrm{~T} \Delta \mathrm{S}<0, \Delta \mathrm{G}<0)$. Moreover, binding is relatively strong with a binding constant of $1.02 \times 10^{5} \pm 0.211 \mathrm{M}^{-1}$ in 1:1 binding mode. In comparison, the ITC data of R8+ and R16+ with MO show the binding to be unspecific clearly suggesting a very weak or no binding process. In addition, NMR studies were conducted to monitor the host-guest binding between 
host P10+ and guest MO. As shown in Figure 5c, the ${ }^{1} \mathrm{H}$ NMR spectra show clear upfield shifts of aromatic signals of $\mathrm{MO}$ as a result of shielding, thus clearly confirming the inclusion complex between P10+ and MO. Host-guest binding between cationic pillararenes and MO has been studied also before in water ${ }^{55}$. The results presented here confirm binding between pillar[5]arene and $\mathrm{MO}$ in physiologically relevant media even though protonation stage of MO has been shown to have influence on the binding mode ${ }^{56}$. In the previously published study, inclusion complex is suggested to form in such a manner that the hydrophobic part of MO is inside the cavity and the sulfonated anionic head is outside the cavity. Based on the DLS and MB displacement assay studies performed in this work, no negative effect of this kind of host-guest binding on hostheparin binding was identified. In conclusion, based on superior host-guest binding results obtained with $\mathbf{P 1 0}+$, further application-relevant studies were continued with only host $\mathbf{P 1 0 +}$. 
Guest: methyl orange (MO)

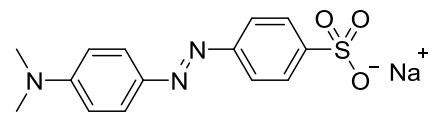

Host: P10+

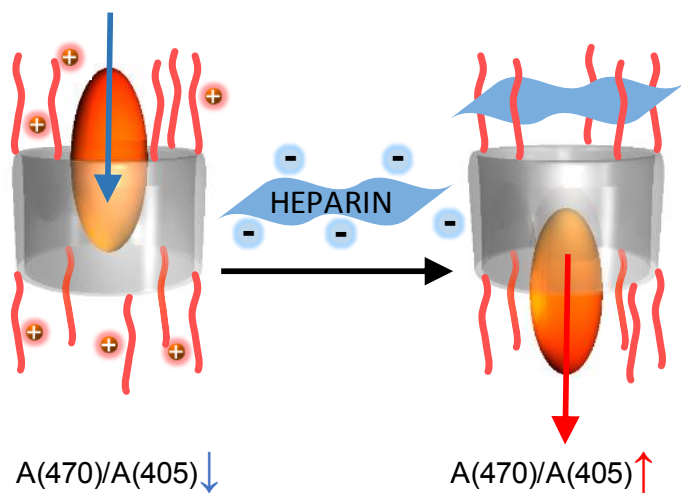

Scheme 2. Structure of the guest methyl orange (MO), and schematic presentation of the hostguest binding between $M O$ and P10+ in addition to host-heparin binding.
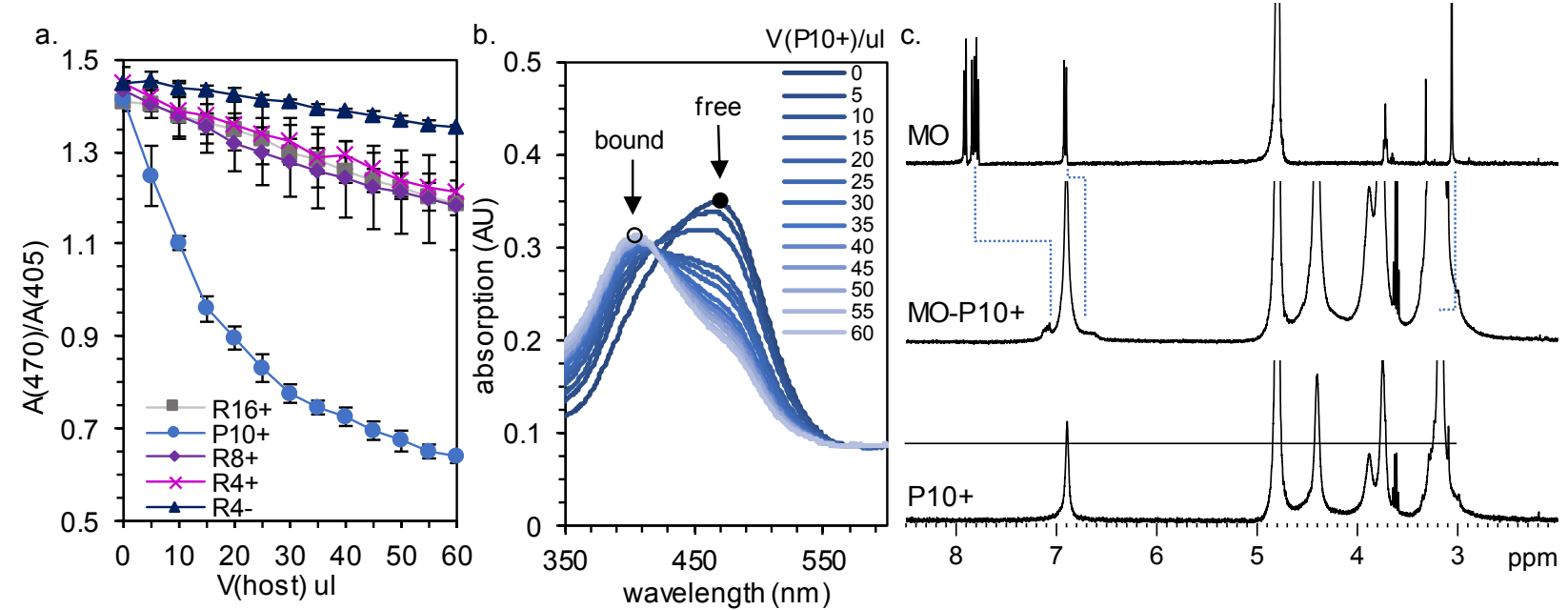

Figure 5. Complexation between the hosts and the guest (MO) was studied with UV-Vis spectroscopy in physiologically relevant Tris-HCl buffer (10 mM tris $150 \mathrm{mM} \mathrm{NaCl}, \mathrm{pH}$ 7.4). a) By comparing absorption maxima of free $(470 \mathrm{~nm})$ and bound $(405 \mathrm{~nm})$ methyl orange, host- 
guest complex formation can be monitored. Inclusion of MO is most pronounce with host P10+. Titrations were carried out in triplicates and averages with standard deviation are presented. b) A blue shift in the absorption spectra is observed when MO solution is titrated with host P10+. c) Host-guest binding was confirmed with ${ }^{1} H$ NMR spectroscopy where clear shift of the aromatic signals of the guest is observed confirming formation of inclusion complex.

In the next step, heparin's effect on the host-guest binding and especially absorption properties of methyl orange was studied. In general, titration of MO-P10+ solution with heparin leads to increase in the $\mathrm{A}(470) / \mathrm{A}(405)$ ratio (Figure 6) indicating that interaction with heparin releases the MO from the host cavity. Analogous MO displacement behavior has also been observed before for a similar triplicate system consisting of cationic resorcinarenes, methyl orange and polyacrylic acid ${ }^{51}$. However, in the system presented here, the host-guest binding likely has more specificity as the pillararene cavity is more confined. In order to optimize our system, heparin titrations were carried out with varying host-guest molar ratios to obtain the ideal combination for heparin concentration monitoring. From the experiments, it was observed that, 1:10 molar ratio of host to guest is ideal as a clear plateau is observed (Figure 6a) unlike with 1:5 ratio, and moreover, further increase of guest concentration to molar ratio of 1:15 did not improve the recovery of $\mathrm{A}(470) / \mathrm{A}(405)$ ratio. The original absorption spectra for all molar ratios are presented in supporting information (Figure $\mathrm{S} 7$ ). From the $\mathrm{A}(470) / \mathrm{A}(405)$ ratio, it is clear that the addition of heparin to the host-guest mixture did not fully release the MO from the host's cavity (Figure 6b). However, correlation between heparin concentration and $\mathrm{A}(470) / \mathrm{A}(405)$ ratio is linear with only minor standard deviations, which is crucial for the signaling system. Highlighting the profitability of our host-guest binding system, the amounts of host and guest are 
reasonable in contrast to a previously published study ${ }^{16}$, based on calix[8]arenes and fluorescent eosin-Y, where nearly 1000 times higher molar ratio of calixarene:eosin-Y was needed for clear signaling.

In general, the host-guest complex needs to be selective towards heparin in order to perform properly in the application-relevant media consisting of competing compounds. Therefore, selectivity of host P10+ towards heparin was studied by comparing it to other glycosaminoglycans. As expected, and based on the high negative charge of heparin, $\mathbf{P 1 0}+$ is most selective towards heparin, as hyaluronic acid and chondroitin sulfate show nearly no competition with heparin (Figure S8). Analogous selectivity profile has been observed also in other previously reported colorimetric ${ }^{11}$ and fluorescent ${ }^{32}$ sensing systems designed for heparin.
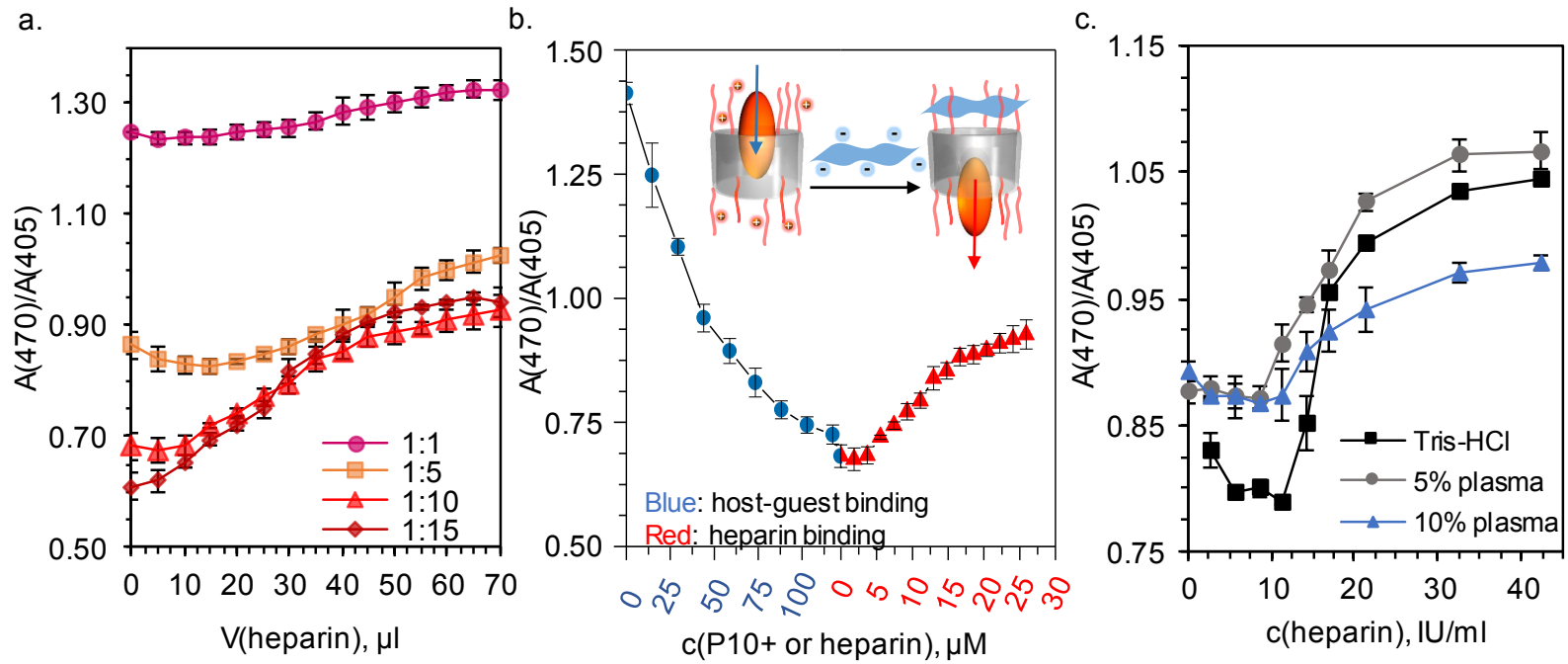

Figure 6. Host-guest complexes of methyl orange $(0.06 \mathrm{mg} / \mathrm{ml})$ and $\mathbf{P 1 0}+(0.42-6.24 \mathrm{mg} / \mathrm{ml})$ with different molar ratios $(1: 1,1: 5,1: 10$ and 1:15) were titrated with heparin to find the most optimal mixture for monitoring heparin levels. Release of MO upon heparin addition was 
observed with UV-Vis spectroscopy and by comparing absorption maxima of free (470 $\mathrm{nm})$ and bound (405 nm) MO. b) By combining data from host-guest titration and heparin titration, it was observed that around $40 \%$ of the $M O$ (from A(470)/A(405) ratio) is recovered upon heparin additions. Blue spheres: MO titrated with host P10+, red triangles: MO-P10+ titrated with heparin. c) Calibration curves for different plasma concentrations was formed by adding $M O$ P10+ solution (1:10 MO-P10+ molar ratio, $4.1 \mathrm{mg} / \mathrm{ml} \boldsymbol{P 1 0}+$ and $0.06 \mathrm{mg} / \mathrm{ml} \mathrm{MO})$ to heparinized samples. Data was recorded after 10 minutes stabilization time. Clearest increase in A(470)/A(405) ratio is observed for Tris-HCl without plasma but $5 \%$ plasma content yields smoothest linearity over largest heparin concentration range. All titrations were carried out in triplicates and averages with standard deviation are presented.

Moreover, it was shown that with this system, a calibration curve for quantitative measurements can be successfully produced, and additionally, the effect of increasing plasma content can be evaluated. Buffer solutions with varying plasma content were heparinized with 2.8-42.4 IU/ml heparin concentration. To these heparinized samples, $30 \mu \mathrm{l}$ of MO-P10+ solution with 1:10 molar ratio $(4.1 \mathrm{mg} / \mathrm{ml} \mathrm{P10+}$ and $0.06 \mathrm{mg} / \mathrm{ml} \mathrm{MO}$ concentration) was added. Absorption spectra stabilized quickly after MO-P10+ addition (Figure S9), and therefore 10 minutes was chosen as the stabilization time for conducting the calibration curve. From Figure 6c, it can be observed that in Tris- $\mathrm{HCl}$ buffer without plasma, variances between different heparin concentrations is the clearest, but the linear region is slightly reduced when compared to $5 \%$ plasma samples, which show a linear dependence between heparin concentration and $\mathrm{A}(470) / \mathrm{A}(405)$ ratio with heparin concentrations ranging from 8.5 to $21.2 \mathrm{IU} / \mathrm{ml}$. For both sample sets, before the linearly increasing region, no response is observed, and after the linear region, a plateau is gradually 
reached. Samples with $10 \%$ plasma suffer from insignificant differences between different heparin concentrations and from the most prominent standard deviations. Similar disruption by increasing plasma concentration in heparin binding has previously been detected in other heparin sensing systems ${ }^{18}$. From the minor standard deviations in 0 and $5 \%$ plasma samples, it can be concluded that large deviations observed in the DLS data do not prevent accurate sensing. To improve the system, for example, binding affinity between heparin and the host could be enhanced, as the system should be operational also at lower application-relevant heparin concentrations $(2-8 \mathrm{IU} / \mathrm{mL}(17-67 \mu \mathrm{M})$ during cardiovascular surgery and $0.2-1.2 \mathrm{IU} / \mathrm{mL}(1.7-$ $10 \mu \mathrm{M})$ in postoperative and long-term care)..$^{57}$ The increased heparin binding affinity could, for instance, be achieved by further enhancing the multivalency or by increasing the sensor concentration. ${ }^{18}$ Binding affinity can also be enhanced by increasing the binding surface

allowing more simultaneous interactions with heparin. ${ }^{15}$ On the other hand, by lowering the hostguest binding affinity, which based on the ITC measurement is high, one could more easily release the guest from the host cavity upon heparin binding.

\section{Conclusions}

In summary, we have shown that macrocyclic hosts are efficient heparin binders, and by introducing a guest molecule to the system, they can be simultaneously utilized also in heparin concentration monitoring. In the heparin neutralization studies, it was observed that multivalency plays a key role when macrocyclic host molecules are used in electrostatic binding of heparin. Based on DLS, methylene blue displacement assay, ITC and anti-Xa studies, binding was efficient in competitive, application-relevant media with both pillar[5]arene and 
resorcin[4]arenes when the molecules had high enough number of positive charges. Interestingly, host P10+ was the best performing host and essentially, it was capable of full heparin neutralization. Additionally, the host-guest complexation does not interfere with the host-heparin binding making the host-guest complex of P10+ and methyl orange an effective heparin binder and sensor. Ratiometric data for heparin concentration monitoring is derived from comparing bound and free absorption maxima of the guest, MO. Furthermore, based on this approach, a calibration curve for quantitative detection of heparin levels in varying amounts of plasma was conducted. We suggest that by further enhancing the sensing abilities at low heparin levels, the host-guest complexes are promising heparin binders and sensors. In conclusion, this study gives a deeper understanding on the possibilities of macrocyclic receptors in signaling system. We envision that based on the thorough solution state measurements, other tertiary supramolecular host-guest-polymer system can be developed, for example, for biomedical applications.

\section{Conflicts of interest}

There are no conflicts to declare.

\section{Acknowledgements}


The authors gratefully acknowledge financial support from the Academy of Finland (grant nos. 263504, 267497, 273645, 286845 and 308578), Aalto University School of Chemical

Engineering, Jane and Aatos Erkko Foundation, Biocentrum Helsinki and the University of

Windsor, ON, Canada. This work was carried out under the Academy of Finland's Centres of Excellence Programme (HYBER 2014-2019). The authors wish to thank Lotta Gustavsson for assisting in the UV-Vis measurements.

\section{References.}

1 B. Casu, A. Naggi and G. Torri, Carbohydr. Res., 2015, 403, 60-68.

2 J. Hirsh, T. E. Warkentin, S. G. Shaughnessy, S. S. Anand, J. L. Halperin, R. Raschke, C. Granger, E. M. Ohman and J. E. Dalen, Chest, 2001, 119, 64S-94S.

3 S. M. Bromfield, E. Wilde and D. K. Smith, Chem. Soc. Rev., 2013, 42, 9184-9195.

4 R. Balhorn, Genome Biol., 2007, 8, 227.

5 J. C. Horrow, Anesth. Analg., 1985, 64, 348-361.

6 S. Wan, J.-L. LeClerc and J.-L. Vincent, Chest, 1997, 112, 676-692.

7 M. Nybo and J. S. Madsen, Basic Clin. Pharmacol. Toxicol., 2008, 103, 192-196.

8 J. W. Vandiver and T. G. Vondracek, Pharmacother. J. Hum. Pharmacol. Drug Ther., 2012, 32, 546-558.

9 S. Kitchen, Br. J. Haematol., 2000, 111, 397-406.

10 M. D. Klein, R. A. Drongowski, R. J. Linhardt and R. S. Langer, Anal. Biochem., 1982, 124, 59-64.

11Z. Zhong and E. V. Anslyn, J. Am. Chem. Soc., 2002, 124, 9014-9015. 
12 K.-Y. Pu and B. Liu, Macromolecules, 2008, 41, 6636-6640.

13 T. Bříza, Z. Kejík, I. Císařová, J. Králová, P. Martásek and V. Král, Chem. Commun., 2008, 0, 1901-1903.

14 S. M. Bromfield, A. Barnard, P. Posocco, M. Fermeglia, S. Pricl and D. K. Smith, J. Am. Chem. Soc., 2013, 135, 2911-2914.

15 A. T. Wright, Z. Zhong and E. V. Anslyn, Angew. Chem. Int. Ed., 2005, 44, 5679-5682.

16T. Mecca, G. M. L. Consoli, C. Geraci, R. L. Spina and F. Cunsolo, Org. Biomol. Chem., 2006, 4, 3763-3768.

17Y. Egawa, R. Hayashida, T. Seki and J. Anzai, Talanta, 2008, 76, 736-741.

18C. W. Chan and D. K. Smith, Chem. Commun., 2016, 52, 3785-3788.

19M. Wang, D. Zhang, G. Zhang and D. Zhu, Chem. Commun., 2008, 0, 4469-4471.

20 S. Wang and Y.-T. Chang, Chem. Commun., 2008, 0, 1173-1175.

21 L. Zeng, P. Wang, H. Zhang, X. Zhuang, Q. Dai and W. Liu, Org. Lett., 2009, 11, 4294-4297.

22Y. Ling, Z. F. Gao, Q. Zhou, N. B. Li and H. Q. Luo, Anal. Chem., 2015, 87, 1575-1581.

23 M. Yang, J. Chen, H. Zhou, W. Li, Y. Wang, J. Li, C. Zhang, C. Zhou and C. Yu, Biosens. Bioelectron., 2016, 75, 404-410.

24R. B. C. Jagt, R. F. Gómez-Biagi and M. Nitz, Angew. Chem. Int. Ed., 2009, 48, 1995-1997.

25 H. Szelke, S. Schübel, J. Harenberg and R. Krämer, Chem. Commun., 2010, 46, 1667-1669.

26M. C.-L. Yeung and V. W.-W. Yam, Chem. - Eur. J., 2011, 17, 11987-11990.

27Q. Chen, Y. Cui, J. Cao and B.-H. Han, Polymer, 2011, 52, 383-390.

28 K.-Y. Pu and B. Liu, Adv. Funct. Mater., 2009, 19, 277-284. 
29 W. Sun, H. Bandmann and T. Schrader, Chem. Eur. J., 2007, 13, 7701-7707.

30 L.-J. Chen, Y.-Y. Ren, N.-W. Wu, B. Sun, J.-Q. Ma, L. Zhang, H. Tan, M. Liu, X. Li and H.B. Yang, J. Am. Chem. Soc., 2015, 137, 11725-11735.

31 J. C. Sauceda, R. M. Duke and M. Nitz, ChemBioChem, 2007, 8, 391-394.

32Y. Ding, L. Shi and H. Wei, Chem. Sci., 2015, 6, 6361-6366.

33 P. Timmerman, W. Verboom and D. N. Reinhoudt, Tetrahedron, 1996, 52, 2663-2704.

34K. Yang, Y. Pei, J. Wen and Z. Pei, Chem. Commun., 2016, 52, 9316-9326.

35X. Ma and Y. Zhao, Chem. Rev., 2015, 115, 7794-7839.

36C. B. Rodell, J. E. Mealy and J. A. Burdick, Bioconjug. Chem., 2015, 26, 2279-2289.

37F. Pan, N. K. Beyeh and K. Rissanen, J. Am. Chem. Soc., 2015, 137, 10406-10413.

38 W. Iwanek and J. Mattay, Liebigs Ann., 1995, 1995, 1463-1466.

39N. K. Beyeh, H. H. Jo, I. Kolesnichenko, F. Pan, E. Kalenius, E. V. Anslyn, R. H. A. Ras and K. Rissanen, J. Org. Chem., 2017, 82, 5198-5203.

40N. K. Beyeh, I. Díez, S. M. Taimoory, D. Meister, A. I. Feig, J. F. Trant, R. H. A. Ras and K. Rissanen, Chem. Sci., 2018, 9, 1358-1367.

41 V. Montes-García, C. Fernández-López, B. Gómez, I. Pérez-Juste, L. García-Río, L. M. LizMarzán, J. Pérez-Juste and I. Pastoriza-Santos, Chem. Eur. J., 2014, 20, 8404-8409.

42 K. Kobayashi, Y. Asakawa, Y. Kato and Y. Aoyama, J. Am. Chem. Soc., 1992, 114, 1030710313.

43 A. S. Michaels, Ind. Eng. Chem., 1965, 57, 32-40.

44 J. van der Gucht, E. Spruijt, M. Lemmers and M. A. Cohen Stuart, J. Colloid Interface Sci., 2011, 361, 407-422. 
45 V. Bagnacani, V. Franceschi, M. Bassi, M. Lomazzi, G. Donofrio, F. Sansone, A. Casnati and R. Ungaro, Nat. Commun., 2013, 4, 1721.

46R. V. Rodik, A. S. Klymchenko, N. Jain, S. I. Miroshnichenko, L. Richert, V. I. Kalchenko and Y. Mély, Chem. Eur. J., 2011, 17, 5526-5538.

47 Capila Ishan and Linhardt Robert J., Angew. Chem. Int. Ed., 2002, 41, 390-412.

48 S. Välimäki, A. Khakalo, A. Ora, L.-S. Johansson, O. J. Rojas and M. A. Kostiainen, Biomacromolecules, 2016, 17, 2891-2900.

49R. A. Shenoi, M. T. Kalathottukaren, R. J. Travers, B. F. L. Lai, A. L. Creagh, D. Lange, K. Yu, M. Weinhart, B. H. Chew, C. Du, D. E. Brooks, C. J. Carter, J. H. Morrissey, C. A. Haynes and J. N. Kizhakkedathu, Sci. Transl. Med., 2014, 6, 260ra150-260ra150.

50M. T. Kalathottukaren, S. Abbina, K. Yu, R. A. Shenoi, A. L. Creagh, C. Haynes and J. N. Kizhakkedathu, Biomacromolecules, 2017, 18, 3343-3358.

51 J. E. Morozova, V. V. Syakaev, A. M. Ermakova, Y. V. Shalaeva, E. K. Kazakova and A. I. Konovalov, Colloids Surf. Physicochem. Eng. Asp., 2015, 481, 400-406.

52 T. Ogoshi, H. Kayama, D. Yamafuji, T. Aoki and T. Yamagishi, Chem. Sci., 2012, 3, 32213226.

36B. Hua, L. Shao, Z. Zhang, J. Sun and J. Yang, Sens. Actuators B Chem., 2017, DOI:10.1016/j.snb.2017.08.141.

54 G. Yu, J. Zhou, J. Shen, G. Tang and F. Huang, Chem. Sci., 2016, 7, 4073-4078.

55 L. S. Yakimova, D. N. Shurpik, L. H. Gilmanova, A. R. Makhmutova, A. Rakhimbekova and I. I. Stoikov, Org. Biomol. Chem., 2016, 14, 4233-4238.

56 S. He, X. Sun and H. Zhang, J. Mol. Struct., 2016, 1107, 182-188.

57 J. Hirsh and R. Raschke, Chest, 2004, 126, 188S-203S. 
TOC

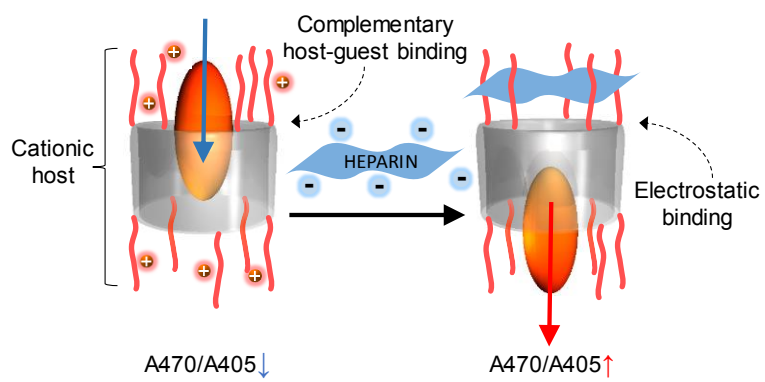

